\title{
RESEARCH METHODS FOR PARAMETERS OF ACCELERATED LOW-ENERGY PROTON BEAM
}

\author{
V.M. Bystritsky ${ }^{a, 1}$, G. N. Dudkin ${ }^{b}$, S. I. Kuznetsov ${ }^{b}$, \\ B. A. Nechaev ${ }^{b}$, V.N.Padalko ${ }^{b}$, A. V.Philippov ${ }^{a}$, \\ A. B. Sadovsky ${ }^{a}$, V.A. Varlachev ${ }^{b}$, O. A. Zvyagintsev ${ }^{b}$ \\ ${ }^{a}$ Joint Institute for Nuclear Research, Dubna \\ ${ }^{b}$ National Research Tomsk Polytechnic University, Tomsk, Russia
}

To study the $p d$-reaction cross section, it is necessary to know the main parameters of the accelerated hydrogen ion beam with a high accuracy. These parameters include the energy ion dispersion, the content of neutrals, and the ratio of atomic and molecular ions of hydrogen in the flux of accelerated particles. This work is aimed at development of techniques and the measurement of the above-mentioned parameters of the low-energy proton beam.

Для изучения сечения $p d$-реакции необходимо знать основные параметры ускоренного пучка ионов водорода с высокой точностью. Эти параметры включают в себя дисперсию энергии иона, содержание нейтральных частиц, отношение атомарных и молекулярных ионов водорода в потоке ускоренных частиц. Данная статья направлена на развитие методов и техники измерения вышеупомянутых параметров протонного пучка низкой энергии.

PACS: 29.27.Fh; 41.75.Ak; 41.85.Qg

\section{INTRODUCTION}

Interest in the study of the nuclear reaction

$$
p+d \rightarrow{ }^{3} \mathrm{He}+\gamma
$$

in the field of ultralow energies during collisions of protons and deuterons $(\mathrm{keV})$ is caused by the possibility of testing theoretical works devoted to solving urgent problems in astrophysics [1-3].

It should be noted that $p d$ reaction is an important astrophysical reaction and a second step of $p p$ cycle that defines the processes of evolution and nucleosynthesis in stars. For stars similar to the Sun, $p p$ cycle is one of the main sources of energy $\sim 98 \%$. Earlier, we have shown for the first time a significant influence of deuteron channeling in crystalline planes of metal deuterated targets made of titanium on the potential of the electronic screening of a nuclear reaction $d(d, n)^{3} \mathrm{He}[4]$.

\footnotetext{
${ }^{1}$ E-mail: bystvm@jinr.ru
} 


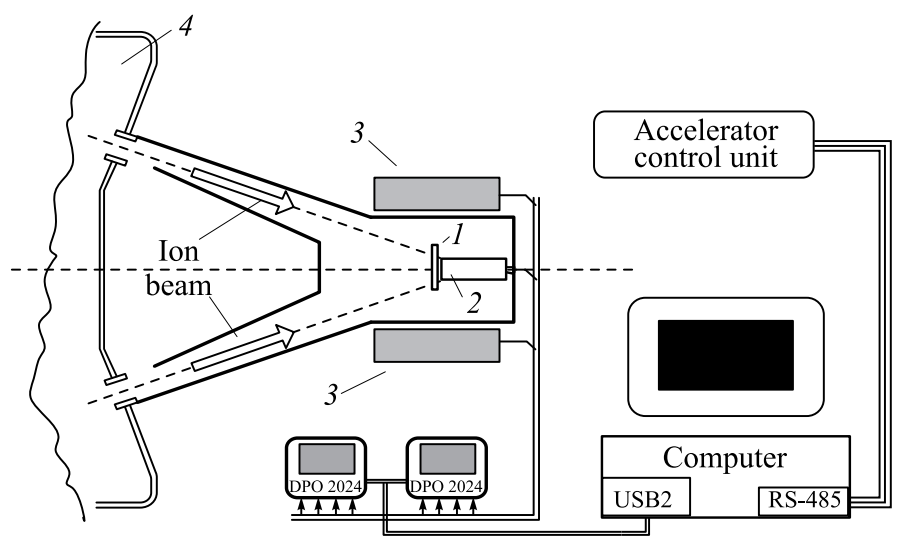

Fig. 1. Scheme of the experiment: 1 - deuterium target; $2-$ multigrid energy spectrometer; $3-$ $\mathrm{NaI}(\mathrm{Tl})$ detectors of $\gamma$ quanta; 4 - Hall ion source (IDM-20)

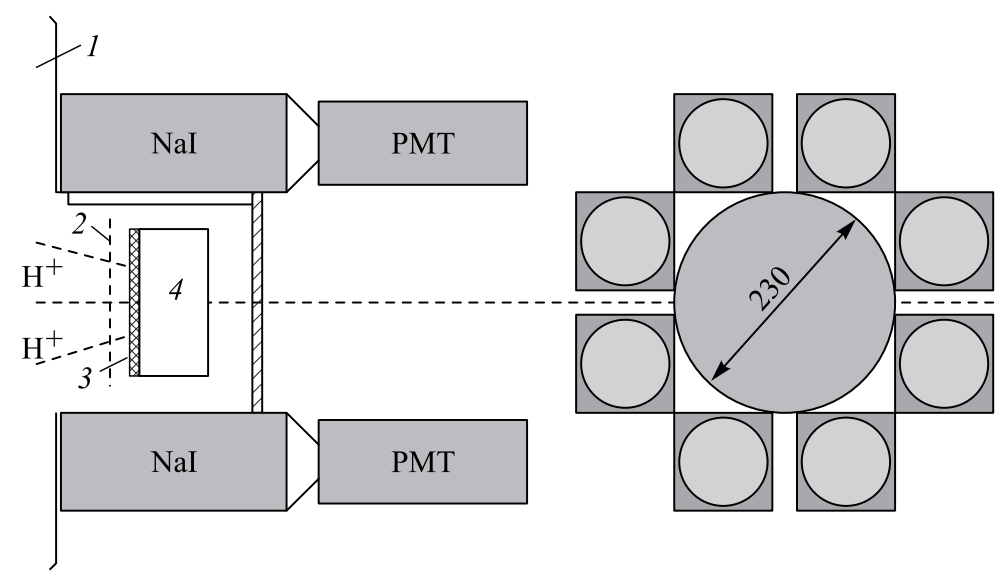

Fig. 2. Location of detectors: 1 - Hall ion source; 2 - grid; 3 - target; 4 - multigrid energy spectrometer

It is possible that for $p d$ reaction the process of proton channeling in crystalline planes of metal deuterated targets influences the potential of screening $U_{e}$. The $p d$ reactions are studied at the installation shown in Figs. 1,2 and described in detail in [5-7].

Hydrogen ion beam, formed as a cylindrical converging cone, falls on the target. The cone-generating angle is $\sim 17.9^{\circ}$ regarding the symmetry axis of the accelerator and is formed by both the geometry of accelerating electric field and the magnetic field generated in the accelerator for magnetic isolation of the cathode [7].

Since the reaction cross section in the field of the Gamow-peak energy $(8-18 \mathrm{keV})$ is very low and is equal to $\sigma_{p d}=10^{-35}-10^{-32} \mathrm{~cm}^{2}$, it is necessary to know the main parameters of the accelerated hydrogen ion beam with a high accuracy. These parameters include the energy ion dispersion, the content of neutrals, and the ratio of atomic and molecular ions of hydrogen in the flux of accelerated particles. This work is aimed at development of techniques and the measurement of the above-mentioned parameters of the low-energy proton beam. 


\section{ENERGY DISPERSION OF HYDROGEN IONS}

To measure the energy spectrum of accelerated protons, a multigrid electrostatic spectrometer was developed which allows us to measure the spectrum of ions by using the target in an operating configuration (Fig. 3).

The developed spectrometer measures the spectrum of protons falling on the entire target area. The target in the operating configuration is used as a collector.

The voltage $150 \mathrm{~V}$ is supplied to the input grid $\mathrm{S} 1$ for cleaning the ion beam from electrons. The reference (input) value of the ion current is measured on the input grid. The voltage $+U$ is supplied to the high-voltage grid S2, which cuts off the ions with energies less than $U \mathrm{eV}$. To provide the symmetry for the geometry of electric field before and after the potential grid S2, it is located (symmetrically) between the same two grounded grids S3. The assembly of the two grids S3 and potential grid S2 are the main analyzing devices. After the analyzing device, the flux of ions passes through the grid S4 and falls on the target $\mathrm{k}$. For correct measurement of the beam current, the voltage $150 \mathrm{~V}$ is supplied to the grid S4 to suppress the secondary ion-electron emission initiated by the ion beam in the material of the collector.

The relative current change in a collector with an increase in voltage on the grid S2 from 0 to full cutoff of ion current gives us the integral (inverted) energy spectrum of accelerated ions.

Several values of accelerating voltage were measured and the energy spectra of accelerated ions were reproduced. In the experiments, we measured the average values of ion currents in the input grid $I_{\mathrm{S} 1}$ and the target $I_{m}$ in each acceleration cycle. The voltage of cutoff $U$ smoothly varied from zero to the value that provides full cutoff of the ion flux falling on the collector (target). Since the current of accelerated ions can vary over a wide range, for the construction of the spectrum we used the value of current in collector normalized to the current value in the input grid $I_{m} / I_{\mathrm{S} 1}$.

In Fig. 4 the numbers indicate: 1 - experimental values, 2 - approximation of the experimental points, 3 - reconstructed energy spectrum.

Energy distribution parameters are average value $\bar{E}=7.65 \mathrm{keV}$ and resolution (full width at half maximum - FWHM) $\Delta E(1 / 2)=17 \%$.

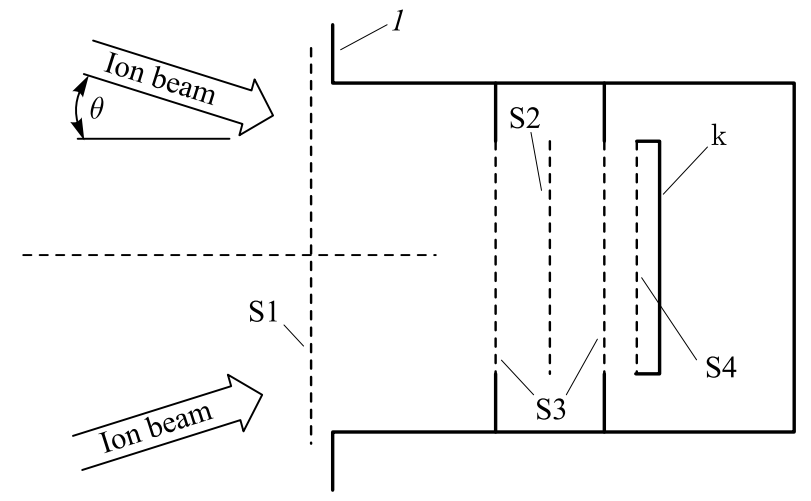

Fig. 3. Electrostatic spectrometer: 1 - vacuum chamber; $\mathrm{S} 1-\mathrm{S} 4-$ metal grids (explanation in text); $\mathrm{k}-$ target 
Divergence of the preset voltage for the acceleration and the center of measured energy distribution can be explained by the fact that the ion beam falls on the target out of the perpendicular. If $\theta$ is the angle between the ion trajectory and the normal to the target, then

$$
\cos (\theta)=\sqrt{\frac{\bar{E}}{E}}
$$

where $\bar{E}$ is the average energy of measured energy distribution and $E$ is the energy of the accelerated ions. The ballistic angle of the conic ion guide $\theta=17.9^{\circ}$.

The change in effective angle during

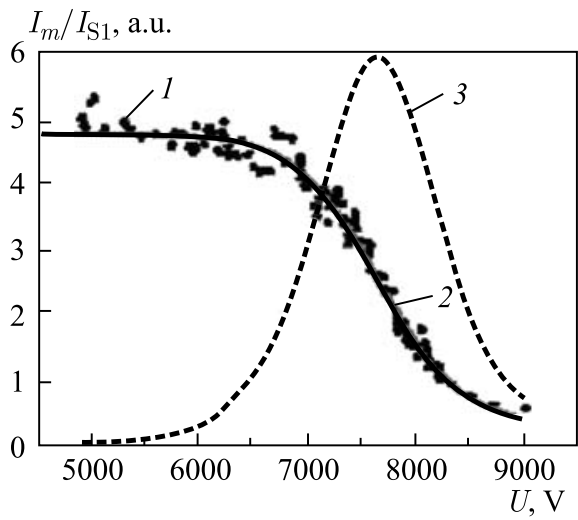

Fig. 4. Current of the target versus the voltage of cutoff for the acceleration voltage of $8 \mathrm{kV}$ falling of ions on the target with the change in their energy can be explained by the focusing action of the magnetic field. For low energies this effect is stronger. At the same time, the trajectory of high-energy ions remains almost unchanged.

The width of the energy distribution of accelerated ions varies slightly with energy. Divergence in estimations is connected with natural instability in the characteristics of the accelerator.

For all energies of the accelerated ions, the value of the energy dispersion can be given: $\Delta E(1 / 2)=(17.4 \pm 0.8) \%$.

\section{MEASUREMENT OF THE NEUTRALIZED HYDROGEN ION FLUX}

To determine the real flux of accelerated particles, it is necessary to take into account the coefficient of secondary electron emission under the action of the charged and fast neutral particles (neutrals) falling on the target.

Fast neutrals can actually be present in the flux of accelerated particles during the charge exchange process. Therefore, we need information on the number of neutrals. The fact of their presence can be detected during secondary electron emission in the interaction of neutrals with the material of the target (collector).

The coefficient of secondary electron emission $\gamma$ is dependent on the material of the collector, the energy of falling particles and their composition, purity of the collector surface, its temperature, pressure and composition of residual gas, etc. To select the reference values of $\gamma$ for the specific conditions of the experiment is practically impossible. It remains to find them experimentally.

To determine the amount of fast neutrals and the value of $\gamma$, we used a grid electrostatic energy analyzer located in front of the target (see Fig. 3).

Note that in the following formulas, to simplify them, the measured value of current in the target is normalized to the current in an input grid $I_{\mathrm{S} 1}$.

Since the coefficients of ion-electron emission are equal for fast ions and neutrals of atomic hydrogen, the current in the metal target $I_{m}$ will be provided by the flux of ions and 
electrons of secondary emission:

$$
I_{m}=I^{+}+\gamma\left(I^{+}+I^{0}\right)
$$

where $I^{+}$is the ion flux and $I^{0}$ is the flux of neutral particles.

When the voltage of ion cutoff is supplied to S2, only neutrals can reach the collector. The current in the target, in this case, is the current of electron emission $I_{e}$ from neutrals:

$$
I_{e}=I_{m}=\gamma I^{0}
$$

When the potential of $150 \mathrm{~V}$ is supplied to $\mathrm{S} 4$, the coefficient of ion-electron emission $\gamma \rightarrow 0$ and, consequently, the recorded current, theoretically, should tend to zero. However, due to the final accuracy of the measuring device (oscilloscope) and parasitical processes (for example, induction), a signal with a final value of $I_{f}$ that is the background for this type of measurement is recorded.

For acceleration voltage of $10 \mathrm{kV}$, we conducted a series of measurements for the quantities $I_{e}$ and $I_{f}$ (Fig. 5).

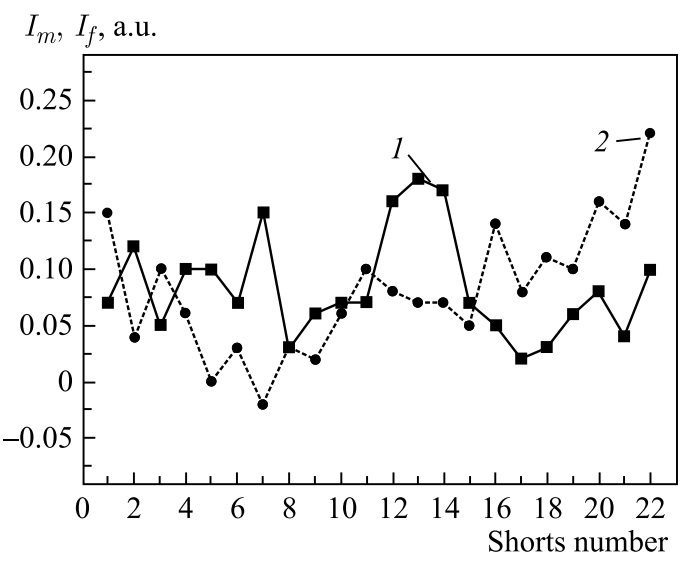

Fig. 5. Current of background measurements (1) and current of electron emission caused by neutral particles (2)

After the data processing we obtained average estimations:

$$
I_{m}=0.081 \pm 0.057, \quad I_{f}=0.084 \pm 0.046 \text {. }
$$

Current of electron emission after subtraction of background is

$$
I_{e}=I_{m}-I_{f}=\gamma I^{0}=-0.003 \pm 0.073 .
$$

It is a fair assumption to say that the neutralized hydrogen ions were not found within the measurement errors. However, to define more exactly the contribution of neutralized particles in a flux of accelerated ions, it is necessary to know the coefficient $\gamma$.

For this purpose, we conducted two similar series of measurements of the ion current (with suppression of the secondary electron emission and without suppression) in the operating mode $U_{\mathrm{S} 2}=0$. 
Neglecting the relatively small contribution of neutrals, we can formulate the expression (3) for these measurements:

$$
I_{m}=I^{+}+\gamma I^{+}\left(U_{\mathrm{S} 4}=0\right), \quad I_{m}^{*}=I^{+}\left(U_{\mathrm{S} 4}=-150 \mathrm{~V}\right) .
$$

From here we find the coefficient of secondary electron emission:

$$
\gamma=I_{m} / I_{m}^{*}-1
$$

In these two series of measurements we obtained the current values:

$$
I_{m}=4.90 \pm 0.11, \quad I_{m}^{*}=2.35 \pm 0.097 .
$$

Then $\gamma=1.085 \pm 0.098$.

Estimating the neutrals from the expression (3) does not differ essentially from the estimating the current of electron emission:

$$
I^{0}=I_{e} / \gamma=-0.003 \pm 0.073
$$

For this energy of hydrogen ions at a voltage of cutoff $U_{\mathrm{S} 2}=0$, the previously measured value of ion current is $I_{m} / I_{\mathrm{S} 1}=6.1$. Then the upper limit for the relative content of fast neutral particles in a flux of accelerated ions is $I^{0} \leqslant 2.4 \%$ with a probability $P=0.95$.

\section{MEASUREMENT OF THE BEAM COMPOSITION OF ACCELERATED HYDROGEN IONS BY THE TIME-OF-FLIGHT METHOD}

If the beam consists of atomic $\mathrm{H}^{+}$and molecular $\mathrm{H}_{2}^{+}$ions of hydrogen, then both types of ions will provide a proportional contribution to the total amount of current. However, the contribution to the reaction yield ${ }^{2} \mathrm{H}(p, \gamma){ }^{3} \mathrm{He}$ will differ significantly for atomic $\mathrm{H}^{+}$and molecular $\mathrm{H}_{2}^{+}$ions of hydrogen, since, in the latter case, the hydrogen ion in a molecule of $\mathrm{H}_{2}^{+}$acquires only half the energy and the reaction cross section decreases rapidly with the decrease in energy. To correctly determine the number of ions that contribute to the reaction, there is a need to apply correction for the number of molecular hydrogen ions.

The ratio of accelerated hydrogen ions $\mathrm{H}^{+}$and $\mathrm{H}_{2}^{+}$in the ion beam of the accelerator IDM-20 was measured by the time-of-flight method. For this purpose, the sufficiently long transport channel $(100 \mathrm{~cm})$ was used for selecting ions with an identical energy but with a different mass. The accelerator generates an ion beam as a series of pulses with a duration of $\sim 2 \mu \mathrm{s}$, which significantly exceeds the difference in times of flight of atomic $\mathrm{H}^{+}$and molecular $\mathrm{H}_{2}^{+}$ions of hydrogen in the transport channel in the energy range of 4-20 keV.

The body of the transport channel (Fig. 6) is a metal tube. The collector for measurement of the ion current is placed at the end of this metal tube. The electrostatic deflection system (modulator) is placed at the inlet of the transport channel.

The positive voltage $V_{m}$ is supplied to a central cylindrical electrode with a diameter $d_{m}=12 \mathrm{~mm}$ and a length $L_{m}=65 \mathrm{~mm}$. The grounded body of the tube with a radius $R=50 \mathrm{~mm}$ is the second electrode of the deflection system.

The metal grid $\mathrm{S}$ with a high transparency is installed in front of the transport channel to measure the parameters of input ion current. The collector is located at a distance $L_{T}=94 \mathrm{~cm}$, and the front face of the modulator is located at a distance of $6 \mathrm{~cm}$ from the input grid. 


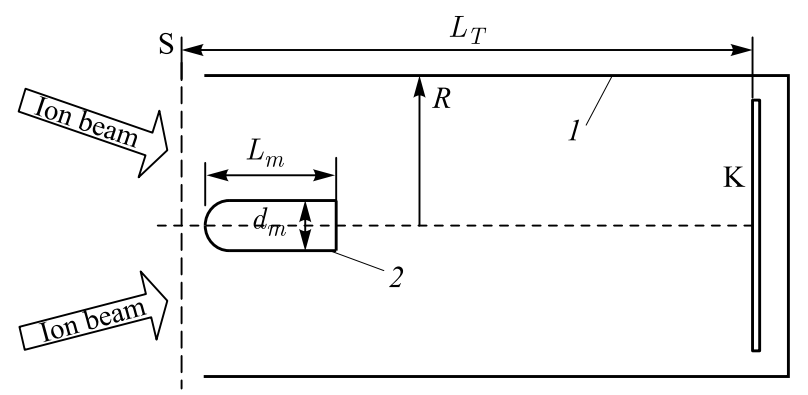

Fig. 6. Scheme of the transport channel: 1 - transport channel; $2-$ modulator; $\mathrm{K}-$ collector; $\mathrm{S}-$ monitor grid

Ballistic focusing implemented in the accelerator generates the ion beam converging on the target. The modulator is located in the transport channel in position of the target. In the absence of the voltage in the modulator, the accelerated ions are deposited on the tube walls. Only a small portion of the ion current reaches the collector. It is assumed that when supplying a positive voltage to the modulator, the ion flux will be focused along the transport channel centerline. The depth of focusing and the required voltage were determined experimentally.

A series of experiments was conducted at a constant voltage of the modulator $V_{m}=$ $+2000 \mathrm{~V}$ and the acceleration voltage of $8 \mathrm{kV}$. At the same time, we recorded oscillograms of the collector current and the grids to build cross correlation functions.

The calculated travel time of ions with an energy of $8 \mathrm{keV}$ from the grid to the collector is equal to $t_{1}=758 \mathrm{~ns}$ for $\mathrm{H}^{+}$and $t_{2}=1070 \mathrm{~ns}$ for $\mathrm{H}_{2}^{+}$. The presence of heavy hydrogen ions in the accelerated beam should lead to the pulse splitting of the collector current. However, only some oscillograms show the significant pulse splitting of the collector current (Fig. 7). The other oscillograms show the pulse stretching. It is rather difficult to estimate the pulse delay time of the collector current relative to the grid current from oscillograms. This information can be obtained from the analysis of correlation functions where there is always one peak, the time coordinate of which is close to the calculated travel time $t_{1}$ of ions $\mathrm{H}^{+}$. The second

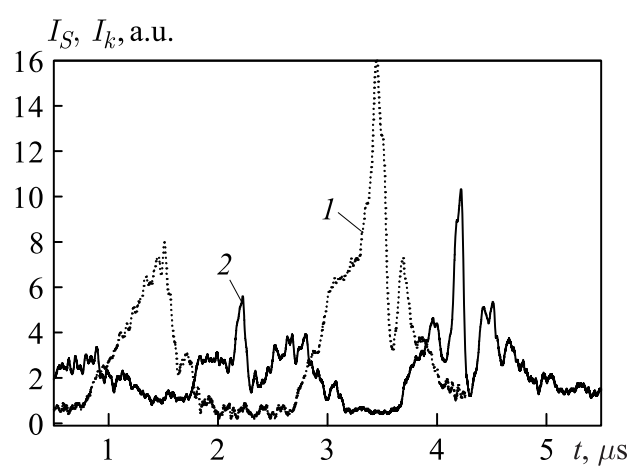

Fig. 7. Fragment of the oscillogram for $V_{m}=$ $2000 \mathrm{~V}: 1$ - grid current $I_{S} ; 2$ - collector current $I_{k}$

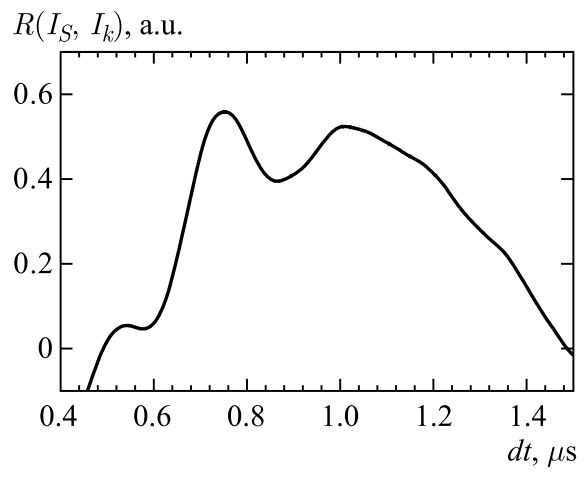

Fig. 8. Correlation function $R\left(I_{S}, I_{k}\right)$ for a fragment of the oscillogram 
peak caused by a delay $t_{2}$ of $\mathrm{H}_{2}^{+}$ions is weaker, which indicates a smaller contribution to the flow of accelerated ions (Fig. 8). The peaks in the graph of the correlation function (Fig. 8) are equal to 755 and $1022 \mathrm{~ns}$ and close to the calculated ones.

Positions of the peaks in the correlation function (Fig. 8) are 755 and 1022 ns. This indicates that there is a contribution of heavy ions $\mathrm{H}_{2}^{+}$to the ion beam. As a result, after the transfer of two types of ion along the transport channel the ion current pulse is amplified. We can numerically estimate the contribution of $\mathrm{H}_{2}^{+}$ions by the degree of current pulse broadening in the collector in comparison with current pulse in the input grid.

For this purpose, we calculate the average amplitude $\bar{A}$ and area $\bar{S}$ for each current pulse in the collector and grid. To be definite, we take the averaging interval from the front of the active pulse to the front of the next pulse. The value $\bar{T}=\bar{S} / \bar{A}$ is the average value of the pulse width and does not depend on the amplitude that can vary within the wide range. If the input flux of ions is monoenergetic, the average value of the pulse width in the grid and collector should be equal $\bar{T}_{S}=\bar{T}_{k}$. Current pulse broadening during the transfer of ions along the transport channel can be caused by their energy dispersion and a lag of the molecular ions $\mathrm{H}_{2}^{+}$. Since the area of the current pulse is proportional to the number of ions in the peak, we can estimate the relative contribution of $\mathrm{H}_{2}^{+}$ions according to the following formula:

$$
\Delta\left(\mathrm{H}_{2}^{+}\right)=\frac{\Delta S}{\bar{S}_{S}}=\frac{\Delta T-\Delta T_{E}}{\bar{T}_{S}},
$$

where $\Delta T=\bar{T}_{k}-\bar{T}_{S}, \Delta T / \bar{T}_{S}$ is the relative pulse broadening due to energy dispersion. In this series of experiments we measured the energy spectra of accelerated hydrogen ions, the energy range width and the corresponding relative pulse broadening $\Delta T / \bar{T}_{S}=0.06$ in the energy range of 8-14 keV.

Using this method we processed a few tens of oscillograms for energies of accelerated ions in the range of $8-17 \mathrm{keV}$. After the data processing we obtained the relative contribution of $\mathrm{H}_{2}^{+}$molecular hydrogen ions in the flux of accelerated ions: $\Delta\left(\mathrm{H}_{2}^{+}\right)=(9.8 \pm 2) \%$ that was taken into account for the processing of the experimental results [8].

\section{CONCLUSIONS}

We have developed a set of tools and a method for measuring the energy spectrum of accelerated ions using a pulsed accelerator IDM-20.

We determined a value of energy dispersion: $\Delta E(1 / 2)=(17.4 \pm 0.8) \%$ for the energy range of accelerated ions of $8-18 \mathrm{keV}$.

We measured the upper limit of the relative content of fast neutral particles in a flux of accelerated ions: $I^{0} \leqslant 2.4 \%$.

The ion contribution of molecular hydrogen $\mathrm{H}_{2}^{+}$was measured in a flux of accelerated ions: $\Delta\left(\mathrm{H}_{2}^{+}\right)=(9.8 \pm 2) \%$.

For fast ions of atomic hydrogen and a metal target used in the experiments, we measured the coefficient of ion-electron emission: $\gamma=1.085 \pm 0.098$.

The work was supported by the Russian Foundation for Basic Research, project No. 14-22-03039. 


\section{REFERENCES}

1. Rolfs C., Rodney W.S. Cauldrons in the Cosmos. Univ. of Chicago Press, 1988.

2. Bachall J. N., Pinsonneault M. H. Standard Solar Models, with and without Helium Diffusion, and the Solar Neutrino Problem // Rev. Mod. Phys. 1992. V. 64. P. 885.

3. Belyaeva V. B. et al. New Proposals for the Investigation of Strong Interaction of Light Nuclei at Super Low Energies // Nucleonika. 1995. V.40. P. 85.

4. Bystritsky V. M. et al. Study of the $p d$ Reaction in the Astrophysical Energy Region Using the Hall Accelerator // Nucl. Instr. Meth. A. 2008. V. 595. P. 543-548.

5. Bystritsky V.M. et al. Channeling Effect in the $d(d, n)^{3} \mathrm{He}$ Reaction on the Titanium Deuteride Crystals // Rus. Phys. J. 2012. V.55, No. 11/2. P. 11-16.

6. Bystritsky V.M. et al. Measurement of Astrophysical $S$-Factors and Electron Screening Potentials for Reaction in $\mathrm{ZrD}_{2}, \mathrm{TiD}_{2}$ and $\mathrm{TaD}_{0.5}$ Targets in the Ultralow Energy Region Using Plasma Accelerator // Nucl. Phys. A. 2012. V. 889. P. 93-104.

7. Bystritsky V.M. et al. Investigation of Parameters and Optimization of Characteristics of Ion Source of Accelerated Particles // Rus. Phys. J. 2012. V.55, No. 11/2. P.51-55.

8. Bystritsky V.M. et al. Study of the $d(p, \gamma)^{3} \mathrm{He}$ Reaction at Ultralow Energies Using a Zirconium Deuteride Target // Nucl. Instr. Meth. A. 2014. V.737. P. 248-252.

Received on November 18, 2014. 